Reprod. Nutr. Dévelop., 1985, 25 (1 B), 335-340.

\title{
Importance spécifique des acides gras polyinsaturés alimentaires de la série n-3 dans l'élaboration des membranes du système nerveux
}

\author{
J. M. BOURRE, C. CHANEZ $\left(^{*}\right)$, O. DUMONT, G. DURAND $\left({ }^{* *}\right), \operatorname{A.~FAIVRE~}\left({ }^{* *}\right)$, \\ A. NOUVELOT $\left({ }^{* * *}\right)$, G. PASCAL $\left({ }^{* *}\right)$, M. PICIOTTI \\ INSERM U-26, Hôpital F.-Widal, 200, rue du Faubourg St-Denis, 75475 Paris Cedex 10. \\ (*) INSERM U-29, Hôpital Port-Royal, 75674 Paris. \\ (**) I.N.R.A., 78350 Jouy-en-Josas. \\ (***) College de France, 75005 Paris.

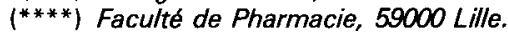

Summary. The specific contribution of dietary polyunsaturated fatty acids (n-3 series) to nervous tissue development.

Polyunsaturated fatty acids, especially $\omega 3$ fatty acids, made a specific contribution to the nervous tissue. Retarded intrauterine growth caused a twofold reduction in the oligodendrocyte ratio of $\omega 3 / \omega 6$ while the same ratio increased by more than twofold in the neurons. A fatty acid-poor diet composed of arachid or sunflower oil dramatically altered the composition of cells and subcellular particles when compared with a soya or rapeseed oil diet containing $\omega 3$ fatty acids; phosphatidylethanolamine was particularly affected. Nerve cell culture showed that brain essential fatty acids are probably docosahexaenoic acid and arachidonic acid.

\section{Introduction.}

Dans le système nerveux, en moyenne, un acide gras sur quatre est polyinsaturé ; ces acides gras participent à la structure des phospholipides mais ils sont absents dans les sphingolipides.

La position $\beta$ des phospholipides est généralement occupée par un acide gras polyinsaturé, qui est le plus souvent le $20: 4 \omega 6$, le $22: 4 \omega 6$, le $22: 5 \omega 3$ et le $22: 6 \omega 3$. Les travaux traitant de l'influence des acides gras polyinsaturés sur la structure et le fonctionnement du système nerveux sont très importants (Morhhauer et Holman, 1963 ; Crawford et Sinclair, 1972 ; Dobbing, 1972 ; Horrocks, 1972 ; Paoletti et Galli, 1972 ; Sinclair et Crawford, 1972 ; Svennerholm et al., 1972 ; Sun et Sun, 1974 ; Dhopeshwarkar, 1975 ; Cook, 1978 ; Tinoco et al., 1979 ; Faivre et al., 1981 ; Loudes et al., 1983 ; Mead et Fulco, 1983 ; Nouvelot et al., 1983b). 
Une carence en acides gras essentiels altère le cours du développement cérébral, période où le besoin est particulièrement important. Le tissu nerveux compensera partiellement ce déficit en synthétisant des acides polyinsaturés en $\omega 9$ qu'il incorpore dans toutes ses membranes.

Toutefois les auteurs ont généralement réalisé des régimes carencés en acides gras des séries $\omega 6$ et $\omega 3$. Or les effets d'une carence spécifique en acides de la série $\omega 3$ (avec apport normal en $\omega 6$ ) sur les cellules du système nerveux sont intéressants. Si un seul des acides essentiels est absent de la nourriture, le cerveau maintiendra constante sa teneur en acides polyinsaturés, mais il y aura un déficit en très longues chaînes qui auraient dû dériver de cet acide absent.

\section{Matériel et méthodes.}

Un lot de rats a été soumis à un des régimes soja ou tournesol dans les conditions précédemment décrites (Bourre et al., 1984). Un autre lot a été nourri avec des huiles d'arachide ou de colza (Primor) (Nouvelot et al., 1983a).

Les fractions cellulaires (neurones, oligodendrocytes, astrocytes) et subcellulaires (myéline, synaptosomes, mitochondries, microsomes) ont été préparées selon les techniques habituelles au laboratoire. Leur pureté a été évaluée par microscopie électronique, dosage d'enzymes marqueurs, analyse de protéines par électrophorèse ou radioimmuno-essai et par étude des lipides complexes spécifiques.

Les cultures de cellules dissociées ont été obtenues à partir d'hémisphères de cerveau fœtal prélevés au $16^{\boldsymbol{e}}$ jour de la vie embryonnaire. Elles ont été cultivées soit en présence d'un milieu chimiquement défini légèrement modifié (Faivre et al., 1981), soit dans un milieu supplémenté avec $10 \%$ de sérum de veau fœetal. Eventuellement, des acides gras ont été ajoutés au milieu synthétique, liés à l'albumine délipidée. Les cultures ont été rincées avec du sérum physiologique, lyophilisées et les lipides extraits avec le mélange chloroforme-méthanol. Les lipides complexes ont été purifiés par chromatographie sur couche mince.

L'hypotrophie intrautérine a été obtenue par clampage d'une artère utérine au $19^{e}$ jour de la gestation.

Les lipides ont été extraits selon la méthode de Folch, éventuellement purifiés par chromatographie sur couche mince pour isoler la phosphatidyléthanolamine. Ces lipides sont méthylés par le trifluorure de bore méthanolique et analysés par chromatographie sur colonne capillaire.

\section{Résultats et discussion.}

\section{a) Hypotrophie intrautérine.}

Bien évidemment, une alimentation quantitativement réduite procure à l'organisme des acides gras polyinsaturés en dose insuffisante. Une réduction du flux sanguin apporte de même au fœtus des acides gras en quantités trop faibles.

Aussi une carence provoquée par la ligature d'une artère utérine qui fait naître des animaux hypotrophiques, détermine des anomalies importantes mais 
opposées dans deux types cellulaires cérébraux (Morand et al., 1982). Le total des acides gras $\omega 6+\omega 3$ reste inchangé comparativement au groupe témoin mais le rapport acides gras $\omega 3$ /acides gras $\omega 6$ est multiplié par plus de deux dans les neurones d'animaux hypotrophiques, alors qu'il est divisé par deux dans les oligodendrocytes. II est intéressant de noter que deux types de cellules peuvent présenter des stigmates opposés après une même agression (tabl. 1).

\section{TABLEAU 1}

Rapport acides gras $\omega 3 /$ acides gras $\omega 6$ dans les lipides totaux des cellules et organites de cerveau d'animaux nourris avec des régimes contenant de l'huile de soja ou de l'huile de tournesol et d'animaux hypotrophiques (âge : 60 jours).

\begin{tabular}{|c|c|c|c|}
\hline \multicolumn{4}{|c|}{$\omega 3 / \omega 6$} \\
\hline & Soja & Tournesol & Hypotrophique \\
\hline Neurones & 0,50 & 0,24 & 0,35 \\
\hline Oligodendrocytes & 0,72 & 0,02 & 0,43 \\
\hline Astrocytes ..... & 0,76 & 0,25 & 0,62 \\
\hline Myéline .. & 0,12 & 0,01 & 0,07 \\
\hline Synaptosomes ... & 0,78 & 0,13 & 0,84 \\
\hline Microsomes & 0,77 & 0.17 & - \\
\hline Mitochondries $\ldots \ldots \ldots \ldots \ldots \ldots \ldots \ldots$ & 0,74 & 0,11 & - \\
\hline
\end{tabular}

b) Animaux nourris avec un régime riche en w3 (huile de soja ou de colza (Primor) et animaux nourris avec un régime pauvre en $\omega 3$ (huile de tournesol et d'arachide).

Nous avons comparé des animaux soumis à un régime normal en acides gras de la série $\omega 6$ mais déficient en acides de la série $\omega 3$ (régime contenant de l'huile de tournesol) avec des animaux soumis à un régime contenant les deux types d'acides (régime contenant de l'huile de soja). Les cellules nerveuses (sauf les neurones) et les organites intracellulaires présentent une quantité totale d'acides gras polyinsaturés identique avec les deux régimes. Mais les cellules et les organites des animaux nourris au tournesol présentent un déficit très important en acide cervonique (22:6) qui est éventuellement compensé par un excès de $22: 5 \omega 6$. Si on compare par exemple des animaux de 60 jours nourris soit avec un régime tournesol, soit avec un régime soja, le rapport $\omega 3 / \omega 6$ est divisé par 16 dans les oligodendrocytes, par 12 dans la myéline, par 2 dans les neurones, par 6 dans les synaptosomes et par 3 dans les astrocytes (tabl. 1). Par contre, les acides gras saturés et monoinsaturés ne sont pratiquement pas altérés (Bourre et al., 1984).

L'importance des acides gras de la série $\omega 3$ est de même bien mise en évidence en étudiant spécifiquement certains phospholipides comme la phosphatidyléthanolamine d'animaux soumis à des régimes arachide ou de colza (Primor) (tabl. 2). Enfin dans nos conditions expérimentales, le $18: 3 \omega 3$ ne semble pas altérer la conversion du $18: 2 \omega 6$ en ses homologues supérieurs $(20: .4 \omega 6$ et $22: 4 \omega 6$ ), seul le taux de $22: 5 \omega 6$ diminue lorsque l'apport en $18: 3 \omega 3$ aug- 
TABLEAU 2

Rapport acides gras $\omega 3$ /acides gras $\omega 6$ dans la phosphatidylethanolamine de divers organites préparés chez des animaux nourris avec des régimes à base d'huile de colza ou d'arachide.

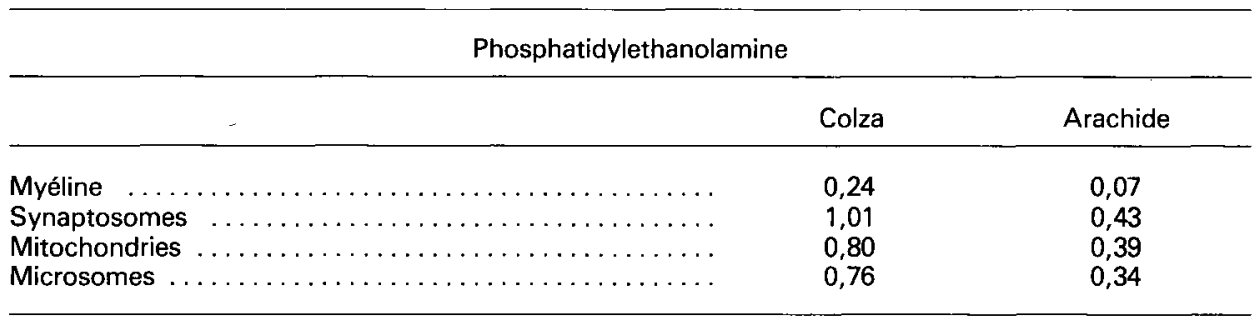

mente. Cette diminution pourrait s'expliquer par une compétition entre le $22: 4 \omega 6$ et le $22: 5 \omega 3$ au niveau de la $\Delta 4$ désaturase. Le $22: 5 \omega 3$ serait alors un substrat préférentiel (Nouvelot et al., 1983a, b). De plus, des expériences préliminaires ont montré que si on réalise des régimes intermédiaires quant à leur contenu en acide linolénique (obtenus en ajoutant au régime arachide des quantités variables et croissantes d'acide linolénique) on observe que dans tous les tissus étudiés (cerveau, foie, rein, testicule), l'apport croissant de $18: 3 \omega 3$ conduit à une augmentation globale des acides gras terminaux de la série $\omega 3$ et inversement une diminution des $\omega 6$ avec toutefois de profondes différences suivant les tissus quant aux variations spécifiques de chaque acide gras. En effet, au niveau du cerveau le taux de $22: 6 \omega 3$ semble croître linéairement pour des apports en $18: 3 \omega 3$ variant de 0,2 à $1,5 \%$ et paraît tendre ensuite vers un palier. Le phénomène inverse est observé pour le $22: 5 \omega 6$. Par contre, les variations au niveau hépatique sont continues mettant en évidence la grande sensibilité hépatique aux apports exogènes (pas de palier). Le comportement particulier du cerveau peut s'expliquer d'une part, par des régulations enzymatiques in situ des séries $\omega 3$ et $\omega 6$, et d'autre part, par une sélectivité de captation au niveau de la barrière hématoencéphalique qui deviendrait prépondérante quand les besoins en acides gras de la série $\omega 3$ sont assurés.

L'étude de la composition des acides gras polyinsaturés des lipoprotéines met en évidence l'importance du métabolisme intra-hépatique intermédiaire dans l'apport en acides gras polyinsaturés au niveau du cerveau; la captation directe par le cerveau des précurseurs $18: 2 \omega 6$ et $18: 3 \omega 3$ est peu importante. Ces précurseurs doivent être allongés et désaturés par le foie en chaînes plus longues, qui sont en fait les acides essentiels pour le cerveau, comme cela semble être montré par les cultures de cellules.

\section{c) Culture de cellules nerveuses.}

En fait le cerveau ne contient pratiquement pas d'acide linoléique ni d'acide linolénique. II est donc possible que ces deux acides soient transformés dans le foie en chaînes plus longues, qui sont en réalité les acides gras essentiels pour le tissu nerveux. Les cellules nerveuses en culture ne sont pas capables de synthétiser des quantités appréciables d'acides docosahexaénoïque. Les cultures de cellules dissociées cultivées en milieu chimiquement défini (dépourvu d'acides gras essen- 
tiels) contiennent deux fois moins d'acides gras polyinsaturés que les cellules dissociées du tissu embryonnaire de départ. Elles contiennent du $20: 3 \omega 9$, preuve que ces cellules sont carencées en acides gras essentiels. La diminution en acides gras polyinsaturés est équilibrée par une augmentation en monoinsaturés, les acides gras saturés n'étant pas affectés. Les acides gras polyinsaturés ajoutés dans le milieu chimiquement défini sont incorporés dans les phospholipides ; l'addition d'acides de la série $\omega 6$ provoque de plus, une augmentation des acides de la série $\omega 3$, ceci étant probablement dû à une meilleure réutilisation des acides gras présents dans les cellules de départ. Après essai de plusieurs combinaisons d'acides gras polyinsaturés, seule l'addition simultanée de $20: 4$ et de $22: 6$ dans le milieu chimiquement défini permet de cultiver des cellules dont le profil en acides gras est similaire à celui du tissu vivant du même âge.

Cette addition stimule la prolifération de petites cellules denses, probablement des oligodendrocytes. Ceci est confirmé par l'étude des acides gras, avec l'apparition de très longues chaînes saturées et monoinsaturées à 22 atomes de carbone, spécifiques de l'oligodendrocyte et de la membrane qu'il synthétise, la myéline.

II apparaît donc que seule l'addition de $20: 4$ et de $22: 6$ dans le milieu de culture de cellules nerveuses permette un meilleur fonctionnement des neurones, mesuré par exemple par l'augmentation de la libération stimulée de neuropeptides (Loudes et al., 1983), la multiplication et la différentiation des oligodendrocytes par le biais d'une composition normale en acides gras de leurs membranes (Bourre et al., 1983).

Les acides gras essentiels pour le cerveau seraient donc les acides de fin de chaîne, synthétisés probablement par le foie à partir des acides linoléique et linolénique.

10e Réunion du groupe Développement I.N.R.A., Rennes, 9-10 mai 1984.

\section{Références}

BOURRE J. M., FAIVRE A., DUMONT O., NOUVELOT A., LOUDES C., PUYMIRAT J., TIXIERVIDAL A., 1983. Effect of polyunsaturated fatty acids on foetal mouse brain cells in culture in a chemically defined medium. J. Neurochem., 41, 1234-1242.

BOURRE J. M., PASCAL G., DURAND G., MASSON M., DUMONT O., PICIOTTI M., 1984. Alterations in the fatty acid composition of rat brain cells (neurons, astrocytes and oligodendrocytes) and of sub-cellular fractions (myelin and synaptosomes) induced by a diet devoid of n-3 fatty acids. J. Neurochem., 43, 342-348.

COOK H. W., 1978. In vitro formation of polyunsaturated fatty acids by desaturation in rat brain : some properties of the enzyme in developing brain and comparison with liver. $J$. Neurochem., 30, 1327-1334.

CRAWFORD M. A., SINCLAIR A. J., 1972. Nutritional influences in the evolution of the mammalian brain. In Lipid, malnutrition and the developing brain. Elsevier, Ciba Found. Symp., pp. 267282.

DHOPESHWARKAR G., 1975. Uptake and transport of fatty acids into the brain and the role of the blood brain barrier system. Adv. Lipid Biochem., 11, 104-142. 
DOBBING J., 1972. Vulnerable periods of brain development. In Lipid, malnutrition and the developing brain. Elsevier, Ciba Found. Symp., pp. 9-23.

FAIVRE A., BAUMAN A., ROSENBAUM E., PUYMIRAT J., GROUSELLE D., TIXIER-VIDAL M., 1981. Differenciation of foetal mouse hypothalamic cells in serum free medium. Dev. Neurosci., 4, 118-129.

HORROCKS L. A., 1972. Ether lipids, 177-272. In Chemistry and biology, Ed. F. SNYDER, Acad. Press.

LOUDES C., FAIVRE A., BARRET A., GROUSSELLE D., PUYMIRAT J., TIXIER-VIDAL M., 1983. Release of immunoreactive TRH inserum free culture of mouse hypothalamic cells. Dev. Brain Res., 9, 231-234.

MEAD J., FULCO A., 1983. The unsaturated and polyunsaturated fatty acids in health and disease. Amer. Lecture, Ser. $\mathrm{n}^{\circ} 5,140-149$.

MOHRHAUER H., HOLMAN R., 1963. Alteration of the fatty acid composition of brain lipids by varying level of dietary essential fatty acids. J. Neurochem., 10, 523-530.

MORAND O., CHANEZ C., MASSON M., DUMONT O., FLEXOR M. A., BAUMANN N., BOURRE J. M., 1982. Alteration in fatty acid composition of neurons, astrocytes, oligodendrocyte, myelin and synaptosomes in intrauterine malnutrition in rat. Ann. Nutr. Metab., 26, 111-120.

NOUVELOT A., DEDONDER-DECOOPMAN E., SEZILLE G., PATURNEAU-JOUAS M., DUMONT O., MASSON M., BOURRE J. M., 1983a. Influence de la teneur en acide linolénique du régime maternel sur la composition en acides gras polyinsaturés des fractions subcellulaires au cours du développement cérébral chez le rat. Ann. Nutr. Metab., 27, 233-241.

NOUVELOT A., BOURRE J. M., SEZILLE G., DEWAILLY P., JAILLARD J., 1983b. Changes in the fatty acid patterns of brain phospholipids during development of rats fed peanut or rapeseed oil, taking into account differences between milk and maternal food. Ann. Nutr. Metab., 27, 173-181.

PAOLETTI R., GALLI C., 1972. Effect of essential fatty acid deficiency on the central nervous system in the growing rat. In Lipid, malnutrition and the developing brain. Elsevier, Ciba Found. Symp., pp. 121-140.

SINCLAIR A. J., CRAWFORD M. A., 1972. The accumulation of arachidonate and docosahexaenoate in the developing rat brain. $J$. Neurochem., 19, 1753-1758.

SUN G., SUN A., 1974. Induction of essential fatty acid deficiency in mouse brain : effects of fats deficient diet upon acid group composition of myelin and synaptosome-rich fractions during development and maturation. Lipids, 9, 450-454.

SVENNERHOLM L., ALLING C., KARLSSON I., SAPIA O., 1972. Effects of offspring of maternal malnutrition. In Lipid, malnutrition and the developing brain. Elsevier, Ciba Found. Symp., pp. 141-157.

TINOCO J., BABCOCO R., HINCENBERG I., MEDWADOWKI B., MILJANICH P., WILLIAMS M., 1979. Linolenic acid deficiency. Lipids, 14, 166-173. 